\title{
On the complexity of detecting hazards*
}

\author{
Balagopal Komarath \\ Saarland University \\ Germany \\ bkomarath@rbgo.in
}

\author{
Nitin Saurabh ${ }^{\dagger}$ \\ Technion - IIT \\ Israel \\ nitinsau@cs.technion.ac.il
}

June 19, 2020

\begin{abstract}
Detecting and eliminating logic hazards in Boolean circuits is a fundamental problem in logic circuit design. We show that there is no $O\left(3^{(1-\epsilon) n}\right.$ poly $\left.(s)\right)$ time algorithm, for any $\epsilon>0$, that detects logic hazards in Boolean circuits of size $s$ on $n$ variables under the assumption that the strong exponential time hypothesis is true. This lower bound holds even when the input circuits are restricted to be formulas of depth four. We also present a polynomial time algorithm for detecting 1-hazards in DNF (or, 0-hazards in CNF) formulas. Since 0-hazards in DNF (or, 1hazards in $\mathrm{CNF}_{\mathrm{N}}$ ) formulas are easy to eliminate, this algorithm can be used to detect whether a given DNF or CNF formula has a hazard in practice.
\end{abstract}

\section{Introduction}

In logic design, one typically extends the Boolean domain $\{0,1\}$ with a third value denoted by ' $u$ ' to indicate the presence of unstable voltage levels. In other words, it indicates that the value of a Boolean variable is unknown. Useful computation can be performed even in the presence of unstable/unknown values. For example, consider a Boolean circuit over $\wedge, \vee$, and $\neg$ gates that takes $2 n$ bits as input and decides whether a majority of the input bits are set to 1. Clearly if at least $n+1$ of its inputs are 1 (or, 0 ) and the rest of the inputs are $u$, the circuit should ideally output 1 (resp., 0 ) 1 However, if the circuit outputs $u$ on such inputs, then the circuit is said to have a hazard. A priori the circuit may be hazard-free or not, but if it is monotone (only $\wedge$ and $\vee$ gates are allowed) then it must be hazard-free [1.

It is well-known that popular physical realizations of the basic logic gates are hazard-free. For example, if we feed a 0 and a $u$ to an AND $(\wedge)$ gate,

\footnotetext{
*https://doi.org/10.1016/j.ipl.2020.105980

${ }^{\dagger}$ This work was done when the author was affiliated with the Max Planck Institute for Informatics, Saarland Informatics Campus, Saarbrücken, Germany.

${ }^{1}$ See Table 1 for the definition of Boolean gates in the presence of $u$.
} 
then the $\wedge$ gate will output a 0 (Table 1). However, it is not necessary that a circuit constructed from hazard-free logic gates is hazard-free. For example, the smallest circuit implementing a one bit multiplexer has a hazard (see, e.g., 1]).

For a logic circuit designer, it is desirable that every circuit they construct is hazard-free. In a recent paper, Ikenmeyer et al. [1] showed that there are $n$-variable Boolean functions with polynomial (in $n$ ) size circuits such that any hazard-free circuit implementation for the same function must use exponentially many gates. Therefore, constructing hazard-free circuits is not always feasible. They also showed that even the computational problem of detecting whether a circuit has a hazard is NP-complete.

Eichelberger's algorithm [2] for detecting hazards in a circuit enumerates all minterms and maxterms of the Boolean function computed by the circuit and evaluates the circuit on each of them. Since an $n$-variable Boolean function can have as many as $\Omega\left(3^{n} / n\right)$ minterms [3] this algorithm is not always efficient. Since this problem is NP-complete, one cannot hope to obtain a polynomial time algorithm that works in general. In such cases, moderately exponential time algorithms are sought over algorithms that employ brute-force search. For example, it is known [4] that the independent set problem has a $O\left(\operatorname{poly}(n) 1.19^{n}\right)$ time algorithm that performs much better than the brute-force $O\left(\operatorname{poly}(n) 2^{n}\right)$ time algorithm. Is it possible to obtain such a moderately exponential time algorithm for hazard detection in circuits?

In this work, we show that the $O\left(3^{n} \operatorname{poly}(s)\right)$ time algorithm for hazard detection on input circuits of size $s$ over $n$ variables is almost optimal under a widely held conjecture known as the strong exponential time hypothesis ( $\mathrm{SETH})$. SETH implies that there is no $O\left(2^{(1-\varepsilon) n}\right.$ poly $\left.(m)\right)$ time algorithm, for any $\varepsilon>0$, for checking whether an $n$-variable, $m$-clause $\mathrm{CNF}$ is satisfiable. We show that there is no $O\left(3^{(1-\varepsilon) n}\right.$ poly $\left.(s)\right)$ time algorithm, for any $\varepsilon>0$, for hazard detection on circuits of size $s$ over $n$ variables assuming SETH. In fact, we show that this is true even when the input circuits are restricted to be formulas of depth four.

We also give a polynomial time algorithm to detect whether a given DNF formula has a 1-hazard. Since 0-hazards in DNF formulas are easy to eliminate, this algorithm can be used to check whether a given DNF formula has a hazard in practice. We remark that, using duality of hazards, this also implies a hazard detection algorithm for CNF formulas.

\section{Preliminaries}

We study Boolean functions $f:\{0,1\}^{n} \rightarrow\{0,1\}$ on $n$ variables where $n$ is an arbitrary natural number. We are interested in Boolean circuits over AND $(\wedge)$, OR $(\vee)$, and NOT $(\neg)$ gates computing such functions. We recall Boolean circuits are directed acyclic graphs with a unique sink node (output gate), where the source nodes (input gates) are labeled by literals, i.e., $x_{i}$ or $\neg x_{i}$ for $i \in[n]$ and non-source nodes are labeled by $\wedge$ or $\vee$ gates. The depth of a gate in the circuit is defined as the maximum number of $\wedge$ or $\vee$ gates occurring on any path from an input gate to this gate (inclusive). (Note that $\neg$ gates do not contribute 
to depth.) The depth of a circuit is then defined to be the depth of the output gate. In particular, CNF and DNF formulas have depth two. We recall formulas are circuits such that the underlying undirected graph is a tree, i.e., every gate other than the output gate has out-degree exactly 1.

We refer to constant depth formulas where all gates of the same depth are of the same type by the sequence of $\wedge$ and $\vee$ starting from the output gate. For example, CNF formulas are $\wedge \vee$ formulas.

In our setting the input variables to circuits are allowed to take an unstable value, denoted by $u$, in addition to the usual stable values 0 and 1 . The truth tables for gates in the basis $\{\wedge, \vee, \neg\}$ in the presence of unstable values are given in Table 1 . The truth table for larger fan-in $\wedge$ and $\vee$ gates in the presence of $u$ can be similarly defined using associativity. Thus, we can evaluate circuits on inputs from $\{u, 0,1\}^{n}$ in the usual inductive fashion.

\begin{tabular}{|c|c|c|c|c|c|c|c|c|c|}
\hline$\wedge$ & $u$ & 1 & 0 & $V$ & $u$ & 0 & 1 & $\neg$ & \\
\hline$u$ & $u$ & $u$ & 0 & $u$ & $u$ & $u$ & 1 & $u$ & $u$ \\
\hline 1 & $u$ & 1 & 0 & 0 & $u$ & 0 & 1 & 0 & 1 \\
\hline 0 & 0 & 0 & 0 & 1 & 1 & 1 & 1 & 1 & 0 \\
\hline
\end{tabular}

Table 1: Truth table for AND, OR, and NOT gates.

We now formally introduce the notion of hazard.

Definition 1. A string $b \in\{0,1\}^{n}$ is called a resolution of a string $a \in$ $\{u, 0,1\}^{n}$ if $b$ can be obtained from a by only changing the unstable values in a to stable values.

For example, strings 0100, 0110, 1110, and 1100 are all possible resolutions of the string $u 1 u 0$, but 0111 is not.

Definition 2. A circuit $C$ implementing a Boolean function has a 1-hazard (or, 0-hazard) on an input $a \in\{u, 0,1\}^{n}$ if and only if $C(a)=u$ yet for all resolutions $b$ of a, the value $C(b)$ is 1 (resp., 0). A circuit has a hazard if it has a 1-hazard or a 0-hazard.

Example 1. Consider the DNF formula $F=\left(x_{1} \wedge x_{2}\right) \vee\left(\neg x_{1} \wedge x_{2}\right) \vee\left(\neg x_{1} \wedge \neg x_{2}\right)$ implementing the function $f$ that evaluates to 0 only when $x_{1}=1$ and $x_{2}=0$. Consider the input $x_{1} x_{2}=0 u$. The function $f$ evaluates to 1 on both resolutions of $0 u$. But, the formula $F$ evaluates to $u$ on input $x_{1}=0, x_{2}=u$. Therefore, $F$ has a 1-hazard at the input $0 u$. We note that $u 1$ is another input where $F$ has a hazard.

We remark that being hazard-free or not is a property of the formula or circuit and not a property of the function being computed by them.

In this paper, we are interested in the time complexity of the following language.

Definition 3. The language HAZARD consists of all circuits that have hazards. 
The hazards in a circuit implementing a function $f$ are closely related to the minterms and maxterms of $f$. A definition of these concepts and their relationship to hazards in circuits follows.

Definition 4. A 1-implicant (0-implicant) of a Boolean function $f$ on variables $x_{1}, \ldots, x_{n}$ is an AND (resp., OR) over a subset I of literals $x_{1}, \ldots, x_{n}, \neg x_{1}, \ldots, \neg x_{n}$ such that for any assignment $a \in\{0,1\}^{n}$, if $I(a)=1$ (resp., $I(a)=0$ ) 2 then $f(a)=1$ (resp., $f(a)=0$ ). In such a case the assignment $a$ is said to be covered by the implicant I. The size of an implicant is defined to be the size of the set $I$.

Example 2. Consider the function $f$ from Example 1. The only 0-implicant of $f$ is $\neg x_{1} \vee x_{2}$ and it is of size 2. The function has five 1-implicants $x_{1} \wedge x_{2}$, $\neg x_{1} \wedge x_{2}, \neg x_{1} \wedge \neg x_{2}, x_{2}$, and $\neg x_{1}$. The assignments $x_{1}=0, x_{2}=0$ and $x_{1}=0$, $x_{2}=1$ are covered by the 1-implicant $\neg x_{1}$.

Definition 5. A 1-implicant (0-implicant) that is minimal with respect to set containment is called a minterm (resp., maxterm).

Example 3. Continuing from Example Q, we note that $f$ has one maxterm $\neg x_{1} \vee x_{2}$ and two minterms, namely $\neg x_{1}$ and $x_{2}$.

We have the following well-known cross-intersection property of the set of all minterms and the set of all maxterms.

Fact 1. Let $S$ be any minterm and $T$ be any maxterm for a function $f$. Then, $S \cap T \neq \emptyset$.

Proof. Suppose not, then there exists an assignment $a$ such that $S(a)=1$ and $T(a)=0$. But then from Definition 4 we have $f(a)=1$ as well as $f(a)=0$, which is a contradiction.

An implicant can be naturally represented as an assignment of variables to $\{u, 0,1\}$ where the variables not in the implicant are set to $u$ and the ones present are set to 0 or 1 so as to make the corresponding literal evaluate to 1 for 1-implicants (or, 0 for 0 -implicants). By evaluating a circuit at a minterm or maxterm, we mean evaluating the circuit on the corresponding assignment in $\{u, 0,1\}^{n}$.

Observation 1. A circuit $C$ implementing a Boolean function $f$ has a 1-hazard (0-hazard) if and only if it has hazard at a minterm (resp., maxterm).

Proof. Given an input $a$ at which $C$ has hazard, consider a minterm or maxterm that covers $a$. It is easily seen that the output of evaluating $C$ on this minterm or maxterm is $u$, because changing stable values in the input to $u$ cannot cause the output to go from $u$ to a stable value.

\footnotetext{
${ }^{2} \mathrm{By} I(a)$, we mean the AND (or, OR) function over the set $I$ of literals evaluated at $a$. We often overload notation to denote both the set of literals in an implicant and the function by the same notation $I$.
} 
Since any minterm or maxterm of an $n$-variable Boolean function can be represented by a string from $\{u, 0,1\}^{n}$, there can be at most $3^{n}$ minterms for a Boolean function. How tight is this upper bound? Chandra and Markowsky [3. gave an improved upper bound of $O\left(3^{n} / \sqrt{n}\right)$ and also gave an example to show that this upper bound is almost tight. We recall the function witnessing this lower bound now. We call it the Chandra-Markowsky (CM) function.

The Chandra-Markowsky (CM) function [3] on $N=3 n$ variables for any natural $n$ is defined as follows: it evaluates to 1 if and only if at least $n$ of the variables are set to 1 and at least $n$ of the variables are set to 0 . This function has $\left(\begin{array}{c}3 n \\ n\end{array}\right)\left(\begin{array}{c}2 n \\ n\end{array}\right)=\Theta\left(3^{N} / N\right)$ minterms. Therefore, it has almost the maximum possible number of minterms.

The Strong Exponential Time Hypothesis (SETH) is a conjecture introduced by Impagliazzo, Paturi and Zane [5, 6] to address the time complexity of the CNF satisfiability problem (CNFSAT). It has been used to establish conditional lower bounds for many NP-complete problems (e.g., 7, 8, ) and problems with polynomial time algorithms (e.g., 9, 10, 11).

Hypothesis 1 (SETH [5, 6]). For every $\varepsilon>0$, there exists an integer $k \geq 3$ such that no algorithm can solve $k$-CNFSAT 3 on $n$ variables in $O\left(2^{(1-\varepsilon) n}\right)$ time.

To establish our lower bound, we reduce from the DNF falsifiability problem (DNFFAlSE): Given a DNF formula as an input, determine whether there exists an assignment that falsifies it.

This problem clearly has the same time complexity as the CNFSAT problem. SETH implies that there is no $O\left(2^{(1-\epsilon) n}\right.$ poly $\left.(s)\right)$ time algorithm ,for any $\epsilon>0$, for DNFFALSE where $n$ is the number of variables and $s$ is the number of clauses.

\section{A tight lower bound}

The idea behind the proof is as follows: The given DNF formula $F$ on $n$ variables has $2^{n}$ assignments. We construct a formula $F^{\prime}$ on $m \sim \log _{3}(2) n$ variables that implements a function with more than $2^{n}$ minterms. This allows us to map each assignment of variables in $F$ to a distinct minterm of the function implemented by $F^{\prime}$. We then show that $F$ is falsifiable by an assignment $a$ if and only if the formula $F^{\prime}$ has a hazard at the minterm $b$ that corresponds to $a$ in the mapping. First, we define the function that is going to be implemented by $F^{\prime}$ and prove some important properties related to it.

Let $s$ be any natural number that is a multiple of 3 . We now define the auxiliary function ACM that will be used in our reduction. It is defined on $s n$ variables which are partitioned into $n$ groups of $s$ variables each. We simply compose the AND function on $n$ variables with the CM function on $s$ variables to define the ACM function. That is,

$$
\operatorname{ACM}\left(X_{1}, \ldots, X_{n}\right)=\operatorname{CM}\left(X_{1}\right) \wedge \cdots \wedge \operatorname{CM}\left(X_{n}\right)
$$

\footnotetext{
${ }^{3}$ Every clause in the $\mathrm{CNF}$ is defined on at most $k$ literals.
} 
where $X_{i}$ denotes the $i$-th group of $s$ variables. We denote the CM function on the $i$-th group of variables $X_{i}$ by $\mathrm{CM}_{i}$.

The following proposition characterizes the minterms and maxterms of the ACM function.

Proposition 2. The following statements are true:

(i) The set of minterms of $\mathrm{ACM}$ is the direct product of the set of minterms of the $n$ disjoint s-variable CM functions.

(ii) The set of maxterms of ACM is given by the union of the set of maxterms of $\mathrm{CM}_{i}$ for $1 \leq i \leq n$.

Proof. (i) We show a one-to-one correspondence between the set of minterms of $\mathrm{ACM}$ and the direct product of the set of minterms of $\mathrm{CM}_{j}$ for $j \in[n]$. For a minterm $I$ of ACM, let $I_{j}$ be the restriction of $I$ to the variables in $X_{j}$ for each $j$. Since ACM evaluates to 1 on $I, \mathrm{CM}_{j}$ must evaluate to 1 on $I_{j}$. Thus, $I_{j}$ is a 1-implicant of $\mathrm{CM}_{j}$ for each $j$. We now argue that in fact it is a minterm. Suppose not, then there exists a $j$ such that $I_{j}$ is not a minterm of $\mathrm{CM}_{j}$. However it must contain a minterm, since it is a 1-implicant. Let $I_{j}^{\prime} \subset I_{j}$ be the minterm contained in $I_{j}$. By replacing the part of $I_{j}$ in $I$ by $I_{j}^{\prime}$ we obtain $I^{\prime}$. Clearly, $I^{\prime} \subset I$ is a 1 -implicant of ACM. Thus, we have a contradiction to the fact that $I$ is a minterm.

On the other hand, given minterms $I_{j}$ of $\mathrm{CM}_{j}$ for each $j \in[n]$, their union $I=\cup_{j \in[n]} I_{j}$ is a minterm of ACM. Suppose not, then there exists $I^{\prime} \subset I$ that is a minterm. Since $I^{\prime} \subset I$, then there exists $j \in[n]$ such that $I_{j}^{\prime} \subset I_{j}$. Thus, we obtain a contradiction to $I_{j}$ being a minterm of $\mathrm{CM}_{j}$.

(ii) For some $j \in[n]$, let $I$ be a maxterm of $\mathrm{CM}_{j}$. Then it is easily seen that $I$ is also a maxterm of ACM. To prove the other direction, for a maxterm $I$ of ACM, we argue that there exists a unique $j \in[n]$ such that $I$ is a maxterm of $\mathrm{CM}_{j}$. Clearly, there exists a $j \in[n]$ such that $I$ is a 0 -implicant of $\mathrm{CM}_{j}$. Since $I$ is a maxterm of ACM, it must only set variables in $X_{j}$. And therefore, the term $I$ is a 0 -implicant of the unique $\mathrm{CM}_{j}$. Hence, the term $I$ must also be a maxterm of $\mathrm{CM}_{j}$.

Huffman 12 showed that for any Boolean function $f$ with the set $\mathcal{M}$ of all minterms, the DNF $F=\bigvee_{I \in \mathcal{M}} I$ is hazard-free. For example, consider the function $f$ defined in Example 1. From Example 3 we know that $\neg x_{1}$ and $x_{2}$ are the only two minterms of it. Therefore, $\left(\neg x_{1}\right) \vee\left(x_{2}\right)$ is the hazard-free DNF implementation for $f$ given by Huffman's construction.

In our reduction, it will be crucial for us to be able to introduce hazards to the implementation at specific minterms. For this purpose we modify Huffman's hazard-free DNF construction as follows. 
Proposition 3. Let $f$ be a function on $n$ variables and $S$ be a set of minterms of $f$ where each minterm in $S$ is of size at most $n-1$. Then, we can construct a DNF for $f$ that has hazards exactly at the minterms in $S$.

Proof. Let $F$ be the hazard-free DNF for $f$ given by Huffman's construction. Let $I$ be a minterm in $S$ and $x$ be a variable not in $I$. Such a variable exists by assumption. Consider the formula $F^{\prime}$ obtained by replacing the term $I$ in $F$ with two new terms, namely $I \wedge x$ and $I \wedge \bar{x}$. $F^{\prime}$ computes the same function and has a 1-hazard at the minterm $I$, since the two new terms evaluate to $u$ on $I$ and every other term will evaluate to 0 or $u$ on $I$. For any minterm not in $S, F^{\prime}$ evaluates to 1 . Therefore, these are the only 1-hazards. Also, $F^{\prime}$ has no 0 -hazards, because every maxterm and minterm intersects contradictorily.

We repeat the aforementioned transformation for every minterm in $S$ to obtain the required DNF for $f$ that has hazards at the minterms in $S$.

To illustrate we consider our running example, the function $f$ from Example 1. We know that $\left(\neg x_{1}\right) \vee\left(x_{2}\right)$ is a hazard-free DNF of $f$. Suppose we want to selectively introduce hazard only at the minterm $\neg x_{1}$. Following Proposition 3 , we modify the hazard-free representation to obtain the following:

$$
\left(\neg x_{1} \wedge x_{2}\right) \vee\left(\neg x_{1} \wedge \neg x_{2}\right) \vee\left(x_{2}\right) .
$$

Suppose we further wanted to introduce hazard at the minterm $x_{2}$. Then, again following Proposition 3, we obtain

$$
\left(\neg x_{1} \wedge x_{2}\right) \vee\left(\neg x_{1} \wedge \neg x_{2}\right) \vee\left(x_{2} \wedge x_{1}\right),
$$

which has hazards at both the minterms. We note that this is the DNF implementation from Example 1 .

The following lemma applies the above construction to the ACM function to efficiently introduce hazards in a selective manner. Notice that any minterm of ACM has some variable that is not in the minterm.

Lemma 4. Consider the ACM function on sn variables where $s$ is regarded as a constant. For $j \in[n]$, let $\mathcal{M}_{j}$ be the set of all minterms of $\mathrm{CM}_{j}$. Further, let $\mathcal{S} \subseteq \mathcal{M}_{i}$ for some $i$. Then, there is a poly-time algorithm that constructs an $\wedge \vee \wedge$ formula for $\mathrm{ACM}$ that has hazards exactly at minterms in the set $\mathcal{M}_{1} \times \cdots \times \mathcal{M}_{i-1} \times \mathcal{S} \times \mathcal{M}_{i+1} \times \cdots \times \mathcal{M}_{n}$.

Proof. Let $F_{j}$ be the hazard-free DNF formula for $\mathrm{CM}_{j}$ and $F_{i}^{\prime}$ be the DNF formula for $\mathrm{CM}_{i}$ that has hazards only at minterms in the set $\mathcal{S}$ obtained by Proposition 3. We output the $\wedge \vee \wedge$ formula $\left(\wedge_{j \neq i} F_{j}\right) \wedge F_{i}^{\prime}$ for ACM. The size of the formula is $O(n)$ because $s$ is a constant.

We now argue that this formula has hazards only at minterms in the set $\mathcal{M}_{1} \times \cdots \times \mathcal{M}_{i-1} \times \mathcal{S} \times \mathcal{M}_{i+1} \times \cdots \times \mathcal{M}_{n}$. Since the individual implementation of $\mathrm{CM}_{j}$ 's have no 0-hazards, by Proposition 2 ( $i i$ ), the formula for ACM has no 0 -hazards. Now suppose $I$ is a minterm of ACM such that the $\wedge \vee \wedge$ formula has a hazard at it. By Proposition $2(i)$, we know that $I_{j}$ is a minterm of $\mathrm{CM}_{j}$ 
for each $j$. Therefore, there exists a $j$ such that the DNF implementation of $\mathrm{CM}_{j}$ has a hazard at $I_{j}$. But then by construction it must be that $j=i$ and $I_{j} \in \mathcal{S}$.

We now prove our main theorem.

Theorem 5. If SETH is true, then for any $\epsilon>0$, there is no algorithm for HAZARD that runs in time $O\left(3^{(1-\epsilon) n}\right.$ poly $\left.(s)\right)$, even when the inputs are formulas of depth four. Here $n$ is the number of variables in the formula and $s$ is the size of the formula.

Proof. Let $r$ be a positive integer and $s=s(r)$ be the minimum integer such that $2^{r} \leq\left(\begin{array}{c}s \\ s / 3\end{array}\right)\left(\begin{array}{c}2 s / 3 \\ s / 3\end{array}\right)$. We will reduce DNFFALSE instances on $r n$ variables to instances of HAZARD on $s n$ variables. In addition, the circuit we output will be an $\vee \wedge \vee \wedge$ formula. Recall, by our choice, $s$ is a multiple of 3 . For any $\epsilon>0$, we claim that there exists a $\delta>0$ such that $3^{(1-\epsilon) s}<2^{(1-\delta) r}$ for sufficiently large $r$. Let $f(s)$ be the number of minterms in the $s$-variable CM function. Then $f(s+3) / f(s) \rightarrow 27$ as $s \rightarrow \infty$. As increasing the number of variables by 3 multiplies the number of assignments by 8 , we have $s(r) / r \rightarrow \log _{27}(8)=\log _{3}(2)$ as $r \rightarrow \infty$. The claim follows.

Let $F$ be the input DNF on $r n$ variables. We consider the variables of $F$ to be partitioned into $n$ groups $Y_{j}, j \in[n]$, of $r$ variables each. We arbitrarily associate with every assignment $\alpha \in\{0,1\}^{r}$ to the variables in $Y_{j}$ a unique minterm $I_{\alpha}$ of $\mathrm{CM}_{j}$ and call this bijection $\beta_{j}$. Recall that $s(r)$ is defined such that the number of minterms of $\mathrm{CM}_{j}$ is at least $2^{r}$. The mapping $\beta_{j}$ is constant-sized and can be computed easily given $j$. The reduction is given in Algorithm 1 It is easy to see that the algorithm runs in polynomial time and produces formulas of depth four. We now argue the correctness of the reduction.

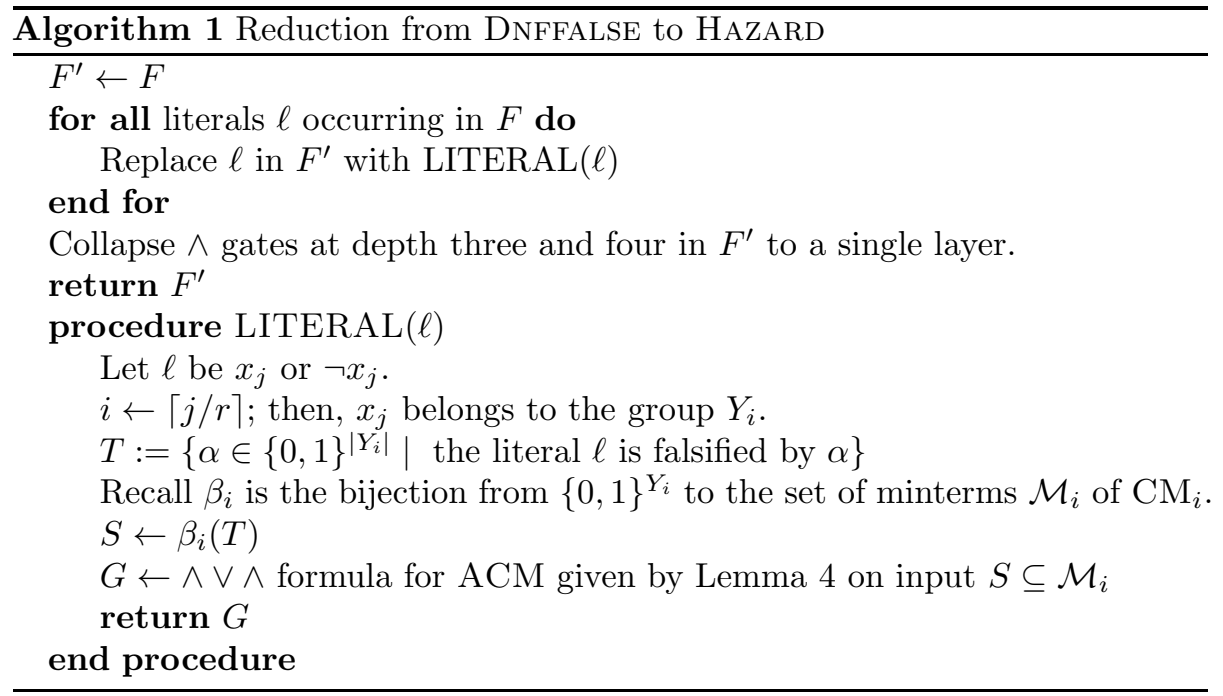


Given an assignment $y \in\{0,1\}^{r n}$ to all variables, let $y_{j}$ denote the restriction of $y$ to variables in the group $Y_{j}$. Further let $I_{y_{j}}$ be the unique minterm of $\mathrm{CM}_{j}$ associated with $y_{j}$. From Proposition $2(i)$, we know that $I_{y}=\mathrm{X}_{j \in[n]} I_{y_{j}}$ is a minterm of ACM. Thus, we associate this minterm $I_{y}$ with the assignment $y$. We will now prove that $F$ is falsified by $y$ if and only if $F^{\prime}$ has a hazard at $I_{y}$.

To prove this, we consider the formula $F^{\prime}$ in the algorithm just before collapsing $\wedge$ gates of depths three and four (i.e., it has depth five). The gates in $F^{\prime}$ correspond to gates in $F$ in the following fashion: The output gate and gates of depth four in $F^{\prime}$ correspond to the output gate and depth one gates in $F$ respectively and the gates of depth three in $F^{\prime}$ correspond to literals in $F$. Since all occurrences of literals in $F$ are replaced with formulas computing $\mathrm{ACM}$ in $F^{\prime}$, the function computed at the output gate and all gates of depth four and three in $F^{\prime}$ is also ACM.

Consider a gate $g$ at depth three in $F^{\prime}$. By construction, the sub-formula rooted at $g$ satisfies the property that it has a hazard at $I_{y}$ if and only if the corresponding literal in $F$ evaluates to 0 on $y$.

We now consider a gate $g$ at depth four in $F^{\prime}$. It is an $\wedge$ gate. Assume that it evaluates to 0 on input $y$ in $F$. Then, at least one of its inputs in $F$ must also evaluate to 0 on $y$. From the above argument about depth three gates, we know that the corresponding gate in $F^{\prime}$ must have a hazard at the minterm $I_{y}$. Therefore, this gate must evaluate to $u$ on $I_{y}$ while the other inputs to the gate $g$ evaluate to 1 or $u$. This is because we are evaluating an implementation of $\mathrm{ACM}$ on one of its minterms. Thus, the sub-formula rooted at $g$ in $F^{\prime}$ must have a hazard at the minterm $I_{y}$ corresponding to $y$. In the other direction, suppose $g$ has a hazard at the minterm $I_{y}$. Then, at least one of its inputs must have a hazard at this minterm, which in turn implies that the corresponding literal in $F$ evaluates to 0 on the assignment $y$. Since $g$ is an $\wedge$ gate we thus obtain that $g$ evaluates to 0 on $y$ in $F$.

Finally we consider the $\vee$ gate $g$ at the root of $F^{\prime}$. If $g$ outputs $u$ on $I_{y}$, all gates feeding into $g$ must output $u$ on $I_{y}$. (They cannot evaluate to 0 because each of them is evaluating ACM function on a minterm.) Therefore, all the corresponding gates in $F$ must output 0 on $y$ causing $F$ to output 0 . On the other hand, if $F$ outputs 0 on $y$, every gate feeding into the root in $F$ must output 0 on $y$ and therefore, all the corresponding gates in $F^{\prime}$ must output $u$ on $I_{y}$ causing $F^{\prime}$ to output $u$ as well.

\section{Detecting hazards in depth-two formulas}

We now look at the time complexity of detecting hazards in depth two formulas. We will focus on input formulas in DNF. The dual statements are true for formulas in CNF. It is known that a DNF formula that does not contain terms with contradictory literals (i.e., $x$ and $\neg x$ for some variable $x$ ) cannot have 0 hazards. Since it is trivial to remove such terms, the interesting case is to detect 1-hazards in DNF formulas. Máté, Das, and Chuang [13 gave an exponential time algorithm that takes as input a DNF formula and outputs an equivalent 
DNF formula that is hazard-free. Such an algorithm is necessarily exponential time because there are functions that have size $s$ DNF formulas such that any hazard-free DNF formulas for it has size at least $3^{s / 3}[3$, Theorem 1.3]. We show that there is a polynomial time algorithm if the goal is to only detect whether an input DNF formula has a 1-hazard.

We start with a crucial observation that help witness 1-hazards in DNF easily. The simplest DNF formula with a 1-hazard is $x \vee \neg x$. We show that every DNF formula with a 1 -hazard has a 1 -hazard $\alpha \in\{0,1, u\}^{n}$ such that when the DNF is restricted by the stable values in $\alpha$, the simplified DNF has the form

$$
x \vee \neg x \vee H,
$$

for some DNF formula $H$ such that no term in $H$ evaluates to 1 . Obviously, it is easy to detect that there is a hazard in such a simplified DNF formulas. We now introduce a definition that will help us formally state the lemma.

Definition 6. A DNF $H$ over variables and constants is said to be equal to 1 if at least one of the terms in it evaluates to 1.

For example, $x \vee \neg x$ is not equal to 1 , though it evaluates to 1 on all possible inputs. On the other hand $x \vee \neg x \vee 1$ is equal to 1 , since it contains the term 1 that trivially evaluates to 1 .

Lemma 6. Let $F$ be a DNF on $n$ variables. Suppose that $F$ has a 1-hazard. Then, there exists $\alpha \in\{0,1, u\}^{n}$ such that $F$ has a 1-hazard at $\alpha$, and furthermore,

$$
\left.F\right|_{\alpha}=x \vee \neg x \vee H,
$$

for some variable $x$ and DNF $H$ that is not equal to 1 . Here, $\left.F\right|_{\alpha}$ represents the DNF obtained by simplifying the terms of $F$ upon substitution of variables by the stable values of $\alpha$. A DNF is simplified by exhaustively applying the following two rules:

(i) Remove terms with a literal that evaluates to 0 ,

(ii) Shorten terms by the removal of literals that evaluate to 1 .

Proof. Let $\beta \in\{0,1, u\}^{n}$ be an arbitrary 1-hazard for $F$. Substitute the stable variables given by $\beta$ in $F$ to obtain a simplified DNF $G$. If $G=x \vee \neg x \vee H$, for some variable $x$ and DNF $H$, then $H$ is not equal to 1 because $G$ must evaluate to $u$ on $\beta$ and, hence, $\beta$ is the required 1-hazard. Suppose not, then either there exists a term of size 1 in $G$ or every term is of size at least 2 . In both cases we construct the required hazard from $\beta$ iteratively. We will increase the number of stable values in $\beta$ at each step while ensuring that it remains a 1-hazard. In particular, we argue in both cases that there exists a variable $x$ in $G$ such that we can set it to 0 and the resulting partial assignment is still a 1-hazard. Clearly this process terminates in at most $n$ steps. We now show how to find the variable in each case. 
Suppose there exists a term of size 1 in $G$. That is, $G=\ell \vee H$, for some literal $\ell$ and, moreover, $H$ does not have $\neg \ell$ as a term. Then we extend the partial assignment $\beta$ by setting $\ell=0$. We now claim that the new partial assignment is still a 1-hazard. This is easily seen because $\ell=0$ either kills a term in $G$ or reduces its size. It never makes a term evaluate to 1 , and therefore the hazard propagates.

In the remaining case, every term in $G$ is of size at least 2 . We pick an arbitrary literal from an arbitrary term and set it to 0. Again as before we can argue that the hazard propagates since any term is either killed or reduced in size, but never evaluated to 1.

Note that if $G$ has only one variable, then it must be $x \vee \neg x$ for some $x$. This completes the proof of the lemma.

We now give a polynomial time algorithm to detect 1-hazards in DNF formulas.

Theorem 7. There is a polynomial time algorithm that detects 1-hazards in DNF formulas.

Proof. Let $F$ be the input DNF formula. From Lemma 6, we know that to check whether $F$ has a 1-hazard it suffices to check for an easy-to-detect hazard. Observe that an easy-to-detect hazard is nothing but a partial assignment $\alpha$ such that $\left.F\right|_{\alpha}$ has both $x$ and $\neg x$ as terms for some variable $x$ and, furthermore, no term evaluates to 1 . In fact, we give a polynomial time procedure to find an easy-to-detect hazard.

To find such a partial assignment we do the following: For every pair of terms $S \wedge x$ and $T \wedge \neg x$ in $F$, for some variable $x$, we check if $S$ and $T$ can be simultaneously set to 1 while no other term in $F$ evaluates to 1 . If so, then clearly this partial assignment is an easy-to-detect hazard.

It is easily seen that the above procedure runs in polynomial time.

Even though all 0-hazards can be eliminated from DNF formulas by removing all terms with contradictory literals, the presence of such terms do not imply a 0 -hazard. For example, the single variable DNF formula $(x \wedge \neg x) \vee x$ does not have any hazards.

In contrast to the poly-time algorithm to detect 1-hazards, the following simple reduction shows that detecting 0-hazards in DNF formulas is hard.

Theorem 8. If SETH is true, then there is no $O\left(2^{(1-\epsilon) n} \operatorname{poly}(s)\right)$ time algorithm, for any $\epsilon>0$, that detects 0-hazards in DNF formulas on $n$ variables and $s$ terms.

Proof. We will reduce the DNF falsifiability problem to this problem. Let $F$ be the input DNF formula for the falsifiability problem. We assume without loss of generality that $F$ does not contain terms with contradictory literals. We now claim that the DNF formula $G=F \vee(x \wedge \neg x)$, where $x$ is a new variable, has a 0 -hazard if and only if $F$ is falsifiable. If $F$ is falsifiable at an input $a$, then the input $(a, u)$ is a 0 -hazard for $G$. On the other hand if $F$ is a tautology, then so is $G$ and, therefore, $G$ cannot have a 0 -hazard. 
The above results can also be stated for CNF formulas using the following observation.

Observation 2. A CNF formula $F$ has a 0-hazard if and only if the DNF formula $G=\neg F$ has a 1-hazard.

Proof. Assume $F$ has a 0 -hazard at $a \in\{u, 0,1\}^{n}$. Each clause in $F$ evaluates to 1 or $u$ on input $a$. Then, the corresponding term in $G$ evaluates to 0 or $u$ respectively which implies that the output of $G$ is also $u$. The other direction is similar.

Observe that any CNF formula that has a 1-hazard contains a clause that contains a variable and its negation. Therefore, we can easily eliminate all 1hazards from a given CNF formula. Combining these observations with theorems for DNF formulas, we have the following theorem.

Theorem 9. There is a polynomial-time algorithm that detects 0-hazards in CNF formulas. Also, if SETH is true, there is no $O\left(2^{(1-\epsilon) n} \operatorname{poly}(s)\right)$ time algorithm for any $\epsilon>0$ that detects 1-hazards in $\mathrm{CNF}$ formulas on $n$ variables and $s$ clauses.

\section{Conclusion}

We show that under SETH the straightforward hazard detection algorithm cannot be significantly improved upon, even when the inputs are restricted to be depth-4 formulas. We also show that there are polynomial time algorithms for detecting 1-hazards in DNF formulas (resp., 0-hazards in CNF), while 0-hazards (resp., 1-hazards) can be easily eliminated. The complexity of hazard detection for depth-3 formulas remain open.

\section{Acknowledgements}

The authors would like to thank the anonymous reviewers. Their suggestions helped to greatly improve the presentation of results in the paper.

\section{References}

[1] C. Ikenmeyer, B. Komarath, C. Lenzen, V. Lysikov, A. Mokhov, K. Sreenivasaiah, On the complexity of hazard-free circuits, in: Proceedings of the 50th Annual ACM SIGACT Symposium on Theory of Computing, STOC 2018, Los Angeles, CA, USA, June 25-29, 2018, 2018, pp. 878-889.

[2] E. B. Eichelberger, Hazard detection in combinational and sequential switching circuits, IBM J. Res. Dev. 9 (2) (1965) 90-99.

[3] A. K. Chandra, G. Markowsky, On the number of prime implicants, Discrete Mathematics 24 (1) (1978) $7-11$. 
[4] M. Xiao, H. Nagamochi, Exact algorithms for maximum independent set, Information and Computation 255 (2017) 126 - 146.

[5] R. Impagliazzo, R. Paturi, On the complexity of k-sat, J. Comput. Syst. Sci. 62 (2) (2001) 367-375.

[6] R. Impagliazzo, R. Paturi, F. Zane, Which problems have strongly exponential complexity?, J. Comput. Syst. Sci. 63 (4) (2001) 512-530.

[7] D. Lokshtanov, D. Marx, S. Saurabh, Lower bounds based on the exponential time hypothesis, Bulletin of the EATCS 105 (2011) 41-72.

[8] M. Cygan, H. Dell, D. Lokshtanov, D. Marx, J. Nederlof, Y. Okamoto, R. Paturi, S. Saurabh, M. Wahlström, On problems as hard as CNF-SAT, in: IEEE Conference on Computational Complexity, IEEE Computer Society, 2012, pp. $74-84$.

[9] K. Bringmann, M. Künnemann, Quadratic conditional lower bounds for string problems and dynamic time warping, in: 2015 IEEE 56th Annual Symposium on Foundations of Computer Science, 2015, pp. 79-97.

[10] A. Abboud, A. Backurs, V. V. Williams, Tight hardness results for LCS and other sequence similarity measures, in: FOCS, IEEE Computer Society, 2015, pp. 59-78.

[11] V. V. Williams, Some open problems in fine-grained complexity, SIGACT News 49 (4) (2018) 29-35.

[12] D. A. Huffman, The design and use of hazard-free switching networks, J. ACM 4 (1) (1957) 47-62.

[13] L. L. Máté, S. Das, H. Y. Chuang, A logic hazard detection and elimination method, Information and Control 26 (4) (1974) $351-368$. 